\title{
Insulinoma: Uma Causa Rara de Hipoglicemia
}

\section{Insulinoma: A Rare Cause of Hypoglycemia}

Cláudia Matta-Coelhoㄹ, Ana Margarida Monteiro ${ }^{1}$, Olinda Marques ${ }^{1}$

Autor Correspondente: Cláudia Matta-Coelho [claudiadmcoelho@gmail.com] Rua das Sete Fontes, 4710-243 Braga, Portugal

\section{RESUMO}

O insulinoma é o tumor neuroendócrino pancreático funcionante mais frequente. A apresentação clínica variável pode mimetizar outras patologias.

Doente do sexo feminino, 61 anos, com vários episódios de alteração do comportamento. Num desses episódios verificou-se hipoglicemia. O estudo etiológico revelou valores inapropriadamente elevados de insulina e peptídeo-C, compatíveis com hiperinsulinismo endógeno. Apesar da tomografia computadorizada, ressonância magnética e cintigrafia dos recetores de somatostatina não revelarem nenhuma lesão, a ecoendoscopia demonstrou uma formação nodular infracentrimétrica pancreática cujo diagnóstico citológico foi concordante com insulinoma. A doente foi submetida a excisão da lesão e mantém-se sem queixas.

Salientamos a apresentação clínica inespecífica e a pequena dimensão da lesão, o que pode levar a um hiato temporal significativo entre o início da sintomatologia e o tratamento.

PALAVRAS-CHAVE: Hipoglicemia/etiologia; Insulinoma 


\begin{abstract}
Insulinoma is the most frequent pancreatic functional neuroendocrine tumour. The clinical presentation is variable and may mimic other diseases.

A 61-year-old female patient, with several episodes of behavioural changes in the last 2 years. In one of these episodes, hypoglycemia was noted. The etiological study revealed inappropriately elevated values of insulin and C-peptide, consistent with endogenous hyperinsulinism. In spite of normal abdominal computed tomography, magnetic resonance and somatostatin receptors scintigraphy, the endoscopic ultrasound showed an infracentimetric pancreatic lesion compatible with insulinoma. The patient was submitted to surgical excision of the lesion, and remains asymptomatic and free of disease.

We emphasize the unspecific clinical presentation and the small dimension of the lesion, which may lead to a significant time gap between the start of the symptoms and the treatment.
\end{abstract}

KEYWORDS: Hypoglycemia/etiology; Insulinoma

\section{INTRODUÇÃO}

O insulinoma é um tumor com origem nas células $\beta$-pancreáticas. Apesar de raro, com uma incidência estimada de quatro casos por milhão/ano, é o tumor neuroendócrino pancreático funcionante mais frequente. ${ }^{1} \mathrm{~A}$ idade média de apresentação ocorre entre a quarta e sexta década, com predomínio ligeiro no sexo feminino. ${ }^{2}$ Os insulinomas são geralmente de pequenas dimensões (inferiores a $2 \mathrm{~cm}$ ), únicos e benignos. Contudo, em cerca de $10 \%$ dos casos, os tumores podem ser malignos, múltiplos e integrar a síndrome de neoplasia endócrina múltipla tipo $1 .{ }^{3}$ Os níveis inapropriadamente elevados de insulina (hiperinsulinismo endógeno) causam hipoglicemia, cujos sintomas são a principal suspeita que conduz ao diagnóstico. $\mathrm{O}$ aumento de peso pode surgir como consequência de pequenas refeições frequentes como forma de evitar os sintomas de hipoglicemia. ${ }^{3}$ As manifestações clínicas de hipoglicemia são inespecíficas, sendo subdivididas em manifestações neuroglicopénicas ou neurogénicas (autonómicas). Os sintomas neurogénicos como taquicardia, tremor e diaforese devem-se à resposta adrenérgica desencadeada pela hipoglicemia, enquanto os sintomas neuroglicopénicos relacionam-se com a privação de glicose a nível cerebral e apresentam um amplo espectro de manifestações, desde fadiga e alterações do comportamento a convulsões ou perda de consciência. A perceção de hipoglicemia advém, principalmente, da sintomatologia neurogénica. ${ }^{4}$ Os sinais/ sintomas ocorrem normalmente em jejum, mas também podem surgir no período pós-prandial, ou em ambos. A hipoglicemia é estabelecida pela tríade de Whipple - sinais e sintomas clínicos de hipoglicemia, glicemia plasmática baixa e melhoria da sintomatologia após a correção da glicemia. Nos doentes não diabéticos, o limite instituído para a hipoglicemia é de $55 \mathrm{mg} / \mathrm{dL} .{ }^{4} \mathrm{Na}$ investigação diagnóstica subsequente de hiperinsulinismo endógeno os valores de insulina e peptídeo-C encontram-se inapropriadamente elevados, superiores a pelo menos $3 \mu \mathrm{U} / \mathrm{mL}$ e 0,6 $\mathrm{ng} / \mathrm{mL}$ respetivamente, quando doseados concomitantemente com uma glicemia plasmática inferior a $55 \mathrm{mg} / \mathrm{dL}$. Caso a tríade de Whipple não possa ser documentada, o doente deve ser submetido a uma prova de jejum prolongado de forma a tentar reproduzir um episódio de hipoglicemia. ${ }^{4}$ Após o diagnóstico bioquímico, segue-se o estudo para localização do tumor realizado por tomografia computadorizada (TC), ressonância magnética (RM), ecoendoscopia ou exames de medicina nuclear. ${ }^{5}$ Em indivíduos com hiperinsulinismo endógeno há outras patologias sem tradução imagiológica e mais raras a considerar, tais como a nesidioblastose e a hipoglicemia autoimune. ${ }^{1}$ No insulinoma, o tratamento preconizado é a exérese cirúrgica do tumor. O tratamento médico é utilizado no controlo pré-operatório da glicemia ou em doentes não candidatos a terapêutica cirúrgica. ${ }^{6}$

\section{CASO CLÍNICO}

Mulher de 61 anos, doméstica. Apresenta antecedentes pessoais de safenectomia em 2007 e síndrome depressiva. Sem antecedentes familiares de relevo. Sem hábitos tabágicos ou etílicos. Medicada com fluoxetina $20 \mathrm{mg} /$ dia e diazepam $5 \mathrm{mg} / \mathrm{dia}$. Nos últimos dois anos, referia vários episódios de sensação de desmaio, de aperto torácico e de tremor involuntário intenso. Segundo a doente, os episódios eram menos frequentes quando realizava várias refeições por dia. Os sintomas surgiam preferencialmente ao acordar e durante a manhã. O estudo analítico, cardíaco e endoscópico gastrointestinal não revelaram alterações. Foi orientada para consulta de Endocrinologia para estudo de possível disfunção endócrina. Na primeira avaliação, a doente apresentava discurso assertivo, mas redundante e lentificado. Ao exame físico, com bom estado geral, peso $65,5 \mathrm{~kg}$, estatura $165 \mathrm{~cm}$ (índice de massa corporal $24,1 \mathrm{~kg} / \mathrm{m}^{2}$ ) e TA $120 / 70 \mathrm{mmHg}$. Apresentava glicemia capilar de $53 \mathrm{mg} / \mathrm{dL}$ com melhoria imediata dos sinto- 


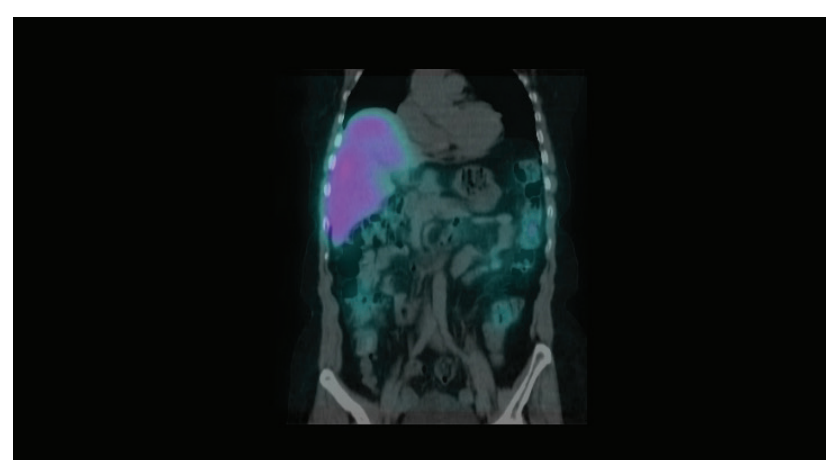

FIGURA 1. Imagem da cintigrafia dos recetores de somatostatina sem focos de captação anómala compatível com a presença de lesões pancreáticas.

mas neuroglicopénicos após correção com açúcar simples. Sem outras alterações de relevo. Negou consumo de sulfonilureias e administração exógena de insulina. Não apresentava cetonúria. Foi aconselhada a evitar consumo de hidratos de carbono simples e foi elaborado um plano alimentar com refeições intervaladas de duas horas, todas com hidratos de carbono complexos. Foi requisitada a avaliação de pesquisa de glicemia capilar regularmente e sempre que ficasse sintomática. Apresentava glicemias em jejum inferiores a 50 mg/dL, duas horas após o pequeno-almoço e ocasionalmente inferiores a $55 \mathrm{mg} / \mathrm{dL}$, valores que foram melhorando progressivamente. A doente aumentou $4 \mathrm{~kg}$ de peso (70 kg), meIhorou das queixas iniciais e não referiu queixas de novo. O estudo analítico, realizado aquando de um episódio de alteração de comportamento, revelou glicemia plasmática de $48 \mathrm{mg} / \mathrm{dL}$, peptídeo C de 1,7 ng/mL (valores de referência: 08-4,2 ng/mL) e insulina de 5,1 $\mathrm{UU} / \mathrm{mL}$ (valores de referência: 2,6-24,9 $\mu \mathrm{U} / \mathrm{mL}$ ), compatível com hiperinsulinismo endógeno. Foi excluído hipotiroidismo ou insuficiência suprarrenal. No estudo etiológico, realizou exames imagiológicos (TC e RM abdominal) que não revelaram alterações. Foi solicitada a ecoendocoscopia que evidenciou uma lesão homogénea, hipoecoica de $6,5 \times 7 \mathrm{~mm}$, de limites bem definidos e regulares na cauda do pâncreas. Procedeu-se a punção aspirativa de agulha fina cuja citologia foi compatível com tumor neuroendócrino. A cintigrafia de recetores de somatostatina demonstrou estudo dentro dos parâmetros da normalidade, sem focos de captação anómala. Após 3 anos desde o início da sintomatologia, foi submetida a excisão cirúrgica da lesão pancreática guiada por ecografia intraoperatória, sem intercorrências peri-operatórias. A histologia foi compatível com tumor neuroendócrino G2 do pâncreas, o índice proliferativo observado (Ki-67) foi de 3,5\%, com pleomorfismo celular moderado, sem necrose. Após a cirurgia, manteve-se assintomática, sem novas queixas sugestivas de hipoglicemia e com registos de glicemia capilar normais. Apresentou uma diminuição de peso, com um peso atual de $67,5 \mathrm{~kg}$. $\bigcirc$ estudo analítico não demonstrou alterações do metabolismo glucídico. Atualmente mantém seguimento na consulta de Endocrinologia.

\section{DISCUSSÃO}

Tal como descrito na literatura, pode haver um atraso significativo no diagnóstico de insulinoma, visto que as manifestações clínicas são inespecíficas e comuns a outras patologias neurológicas/psiquiátricas. ${ }^{7}$ Salientamos a clínica apresentada e o facto da doente referir que conseguiu durante muito tempo evitar a sintomatologia com a ingestão de várias refeições diárias. Neste caso foi possível a avaliação simultânea de glicemia plasmática, insulina e peptídeo-C num episódio de hipoglicemia espontânea, não sendo necessário realizar a prova de jejum prolongado, considerado o "gold standard" no diagnóstico de hipoglicemia. ${ }^{8}$ A sensibilidade dos exames imagiológicos na deteção dos insulinomas é variável e dependente das dimensões do tumor, que são frequentemente muito reduzidas. Atualmente, não há consenso sobre o melhor método de localização. A TC e a RM são as primeiras a ser utilizadas, já que são técnicas não invasivas, com uma sensibilidade de 33\% - 64\% e de $40 \%$ - 90\% respetivamente. ${ }^{9}$ Relativamente à cintigrafia dos recetores de somatostatina a sensibilidade descrita é de $50 \%$ - 70\%. ${ }^{5}$ O passo seguinte é o recurso a uma técnica mais invasiva e operador-dependente como a ecoendocoscopia cuja sensibilidade é entre 86,6\% - 92,3\%. ${ }^{9}$ Nos casos em que a intervenção cirúrgica não é possível ou a localização do tumor é muito demorada está indicado fazer terapêutica médica e/ou nutricional para minimizar as crises e os riscos consequentes. A terapêutica médica utilizada em casos de insucesso da modificação do plano alimentar, é o diazóxido que inibe diretamente a secreção de insulina e pode controlar a hipoglicemia em 50\% - 60\% dos doentes. Além do diazóxido são alternativas médicas, em doentes não candidatos a cirurgia, os análogos da somatostatina, nomeadamente o octreótido e o lanreótido, e a ablação da lesão com etanol. ${ }^{8}$ Em tumores benignos, após ressecção cirúrgica, a taxa de recorrência é baixa, cerca de 5\%, e não há uma alteração significativa da sobrevida. ${ }^{10}$

CONFLITOS DE INTERESSE: Os autores declaram não ter qualquer conflito de interesse na realização do presente trabalho.

FONTES DE FINANCIAMENTO: Não houve qualquer fonte de financiamento na realização do presente trabalho. 
CONFIDENCIALIDADE DOS DADOS: Os autores declaram ter seguido os protocolos da sua instituição acerca da publicação dos dados de doentes.

PROTEÇÃO DE PESSOAS E ANIMAIS: Os autores declaram que os procedimentos seguidos na elaboração do presente trabalho estão em conformidade com as normas das comissões de investigação clínica e de ética, bem como da declaração de Helsínquia e da Associação Médica Mundial.

CONFLICTS OF INTEREST: The authors declare that they have no conflicts of interest. FINANCIAL SUPPORT: This work has not received any contribution, grant or scholarship.

CONFIDENTIALITY OF DATA: The authors declare that they have followed the protocols of their work center on the publication of data from patients.

PROTECTION OF HUMAN AND ANIMAL SUBJECTS: The authors declare that the procedures followed were in accordance with the regulations of the relevant clinical research ethics committee and with those of the Code of Ethics of the World Medical Association (Declaration of Helsinki).

\section{REFERÊNCIAS}

1. Kittah NE, Vella A. Management of endocrine disease: Pathogenesis and management of hypoglycemia. Eur J Endocrinol. 2017:EJE-16-1062.

2. Masharani U, Gitelman S. Hypoglycemic Disorders. In: Gardner D, Shoback D, editors. Greenspan's Basic and Clinical Endocrinology. $9^{\text {th }}$ ed. Washington: Mc Graw Hill Lange; 2011. p. 663-7.

3. Iglesias P, Díez JJ. Management of endocrine disease: A clinical update on tumor-induced hypoglycemia. Eur J Endocrinol. 2014;170: R147-57.

4. Cryer PE, Axelrod L, Grossman AB, Heller SR, Montori VM, Seaquist ER, et al. Evaluation and management of adult hypoglycemic disorders: An endocrine society clinical practice guideline. J Clin Endocrinol Metab. 2009;94+-:709-28.

5. Tamm E, Bhosale P, Lee J, Rohren E. State-of-the-art imaging of pancreatic neuroendocrine tumors. Surg Oncol Clin N Am. 2016;25:375-400.

6. Mehrabi A, Fischer L, Hafezi M, Dirlewanger A, Grenacher L, Diener MK, et al. A systematic review of localization, surgical treatment options, and outcome of insulinoma. Pancreas. 2014:43:675-86.

7. Caldas AR, Teixeira S, Giestas A, Amaral C, Cardoso MH. Insulinoma pancreático: casuística de um hospital central e revisão da literatura. Rev Port Endocrinol Diabetes Metab. 2015;11:181-7

8. Ito T, Igarashi H, Jensen RT. Pancreatic neuroendocrine tumors: Clinical features, diagnosis and medical treatment: Advances. Best Pract Res Clin Gastroenterol. 2012;26:737-53.

9. Okabayashi T, Shima Y, Sumiyoshi T, Kozuki A, Ito S, Ogawa Y, et al. Diagnosis and management of insulinoma. World J Gastroenterol. 2013;19:829-37.

10. Tsang Y-P. Lang BH-H. Shek TW. Assessing the short- and long-term outcomes after resection of bening insulinoma. ANZ J Surg. 2014;86:706-10. 